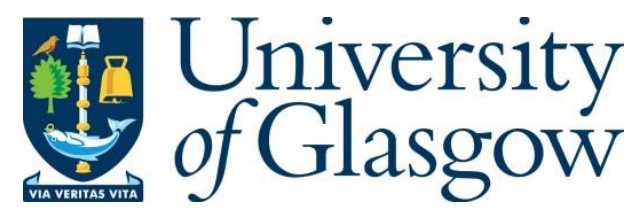

Kelp, C. (2019) Inquiry and the transmission of knowledge. Philosophy and Phenomenological Research, 99(2), pp. 298-310.

There may be differences between this version and the published version. You are advised to consult the publisher's version if you wish to cite from it.

This is the peer reviewed version of the following article Kelp, C. (2019) Inquiry and the transmission of knowledge. Philosophy and Phenomenological Research, 99(2), pp. 298-310, which has been published in final form at http://dx.doi.org/10.1111/phpr.12489. This article may be used for non-commercial purposes in accordance with Wiley Terms and Conditions for Self-Archiving.

http://eprints.gla.ac.uk/140981/

Deposited on: 16 May 2017

Enlighten - Research publications by members of the University of Glasgow http://eprints.gla.ac.uk 


\title{
Inquiry and the Transmission of Knowledge
}

\author{
Christoph Kelp*
}

1. Many think that competent deduction is a way of extending one's knowledge. In particular, they think that the following captures this thought at least roughly:

Transmission- $K$. If $(\mathrm{C} 1)$ one knows that $p,(\mathrm{C} 2)$ one competently deduces $q$ from $p$, and (C3) one comes to believe that $q$ based on one's deduction, then $(\mathrm{C} 4)$ one thereby comes to know that $q{ }^{1}$

Others disagree. They argue that cases like the following cause trouble for Transmission-K:

Zebra. You are at the zoo. Currently you are standing in front of the zebra enclosure and see a black-and-white striped equine creature inside. Since you can tell a zebra from the way it looks, you come to know the proposition that $\mathrm{Z}$ $=$ it is a zebra $(\mathrm{C} 1)$. From this you competently deduce the proposition that $\neg \mathrm{CDM}=$ it is not a cleverly disguised mule (C2) and you thereupon come to believe $\neg$ CDM (C3) [Dretske 1970].

What foes of Transmission- $K$ typically point out at this stage is that, intuitively, your belief that $\neg \mathrm{CDM}$ does not qualify as knowledge. Given that this intuition is correct, Transmission- $K$ fails. After all, as the description of the case clearly indicates, you satisfy all three conditions in the antecedent of the principle. However, since you don't know what you come to believe, you do not satisfy the consequent.

Even if it is intuitive that you do not come to know that $\neg \mathrm{CDM}$ in Zebra, Transmission- $K$ is also highly intuitive [e.g. Williamson 2000]. This raises the question whether we should accept the counterexamples and reject Transmission- $K$ or else hold on to Transmission- $K$ and embrace that agents in cases like Zebra do acquire knowledge. One key aim of this paper is to argue that we should indeed reject Transmission- $K(\S \S 2,3)$. Crucially, the argument I will offer is theoretical in the sense that it relies on a number of independently plausible premises to establish the

${ }^{*}$ Institute of Philosophy, KU Leuven, Kardinaal Mercierplein 2, BE-3000 Leuven, Email: christoph.kelp@hiw.kuleuven.be

1 [Williamson 2000, Hawthorne 2004]. This principle also sometimes goes by the name of Closure. In contrast with the debate on knowledge, the literature on warrant distinguishes sharply between closure principles and transmission principles. Since the above would clearly be categorised as a transmission principle there, I decided to go with the label Transmission-K. 
failure of Transmission- $K$, rather than just on the intuition of absence of knowledge in cases like Zebra. What's more, the argument also ushers the way toward carving out a certain type of case in which Transmission- $K$ fails ( $\$ 4)$.

If Transmission- $K$ fails, these failures should be borne out by the correct account of knowledge. The second aim of this paper is to argue that a certain kind of virtue epistemological account of knowledge [e.g. Author 2013, 2016, Greco 2010, 2012, Sosa 2011, 2015] can do just that (\$§5-8). To be more precise, I will show that this kind of account has the resources to accommodate not only that Transmission- $K$ fails in the relevant type of case, but also that all remaining instances of Transmission- $K$ continue to hold. In this way, the virtue epistemological account of knowledge not only bears out failures of Transmission- $K$ but also supports a suitably restricted version of the principle.

Finally, I will compare the virtue epistemological approach to Transmission$K$ with the most prominent alternative, which explains failures of transmission in terms of a sensitivity account of knowledge. I will argue that the virtue epistemological approach is preferable as only it can predict failures of transmission just where it ought to $(\S \S 9-11)$.

2. This section offers an argument against Transmission-K. In order to achieve this, I want to focus on inquiry and, more specifically, on inquiry into specific whether questions.

Inquiry is a goal-directed activity. As such, it has a success condition, a condition under which its goal is attained. What is the goal of inquiry? Or, to be more precise, what is the goal of an agent's inquiry into whether $p$ ? One answer, which I take to be platitudinous, is that one's inquiry into whether $p$ aims at properly settling for oneself the question whether $p$ (henceforth just settling the question whether $p$ for short). ${ }^{2}$ Correlatively, we get:

Inquiry Success. If one's inquiry into whether $p$ is successful, then it settles the question whether $p$.

Another thesis that I take to be platitudinous is that question-begging excludes question settling. This gives us:

No Settling. If one's inquiry into whether $p$ is question-begging, then it doesn't settle the question whether $p$.

Now consider the following two cases involving inquiry into whether $\neg \mathrm{CDM}$ :

Inquiry 1. You are at the zoo. Currently you are standing in front of the zebra enclosure and see a black-and-white striped equine creature inside. Since you

\footnotetext{
${ }^{2}$ Note that this fits nicely with Pamela Hieronymi's view of belief that $p$ as (positively) settling for oneself the question whether $p$ [Hieronymi 2009: 139], which will give us the independently plausible result that the goal of inquiry is a species of belief. Note, however, that it does not follow that belief is the goal of inquiry. After all, inquiry aims at properly settling for oneself the question whether $p$, not just at settling it for oneself. Rather, what emerges is the attractive view that inquiry aims at belief that is proper in some sense to be specified.
} 
can tell a zebra from the way it looks, you come to know that $Z$. You now want to find out whether $\neg \mathrm{CDM}$. To settle this question you take a hair sample from the animal and perform a DNA analysis. The result of the analysis is that the animal is indeed a zebra. From this, you competently deduce and thereupon come to believe that $\neg \mathrm{CDM}$.

Inquiry 2. You are at the zoo. Currently you are standing in front of the zebra enclosure and see a black-and-white striped equine creature inside. Since you can tell a zebra from the way it looks, you come to know that $\mathrm{Z}$. You now want to find out whether $\neg \mathrm{CDM}$. To settle this question you exploit the entailment from $\mathrm{Z}$ to $\neg \mathrm{CDM}$ to competently deduce and thereupon come to believe that $\neg \mathrm{CDM}$.

There is a clear difference between Inquiry 1 and 2. While there is nothing wrong with your inquiry in Inquiry 1, in Inquiry 2, your inquiry is problematic. What explains this difference? The by far best answer that I can think of is that, in Inquiry 2 , as opposed to 1 , your inquiry is question-begging (more on this in $\S 3$ below). Reflection on the difference between Inquiry 1 and 2 thus motivates the following thesis:

Question-Begging. In Inquiry 2 , your inquiry into whether $\neg \mathrm{CDM}$ is questionbegging.

Now, one might wonder what all of these rather unexceptional points have to do with Transmission- $K$. To see the answer, note that there is a relation between knowledge and the goal of inquiry/success in inquiry. While inquiry into whether $p$ can platitudinously be characterised as aiming at settling the question whether $p$, there is an ongoing debate over how one might characterise the goal of inquiry in a more substantive fashion. There are a number live options in the literature: according to some knowledge is the goal of inquiry [e.g. Author 2014, Millar 2011, Williamson 2000], while others hold that it's justified belief [e.g. Davidson 2005, Feldman 2002, Rorty 1995] or true belief [e.g. Kvanvig 2003, Lynch 2005]. Fortunately, for present purposes, the issue doesn't have to be decided. The reason for this is that knowledge entails justified true belief. Given that all live candidates for the goal of inquiry are entailed by knowledge, there is reason to believe that knowledge is at least sufficient for attaining success in inquiry. In other words,

Knowledge Sufficiency. If one's inquiry into whether $p$ leads one to knowledge that $p$ (not- $p$ ), then one's inquiry into whether $p$ is successful.

It is easy to see that from Inquiry Success, Question-Begging, No Settling and Knowledge Sufficiency, it follows that, in Inquiry 2, your inquiry into $\neg$ CDM does not lead you to knowledge that $\neg \mathrm{CDM}$. But now recall how your inquiry in Inquiry 2

\footnotetext{
${ }^{3}$ It may be worth noting that even champions of Transmission- $K$ and the related transmission of warrant principle (see $\$ 3$ below) acknowledge this point [e.g. Pryor 2000, Markie 2005, Pritchard 2007].
} 
proceeds: from $\mathrm{Z}$, which you know, you competently deduce and thereupon come to believe that $\neg$ CDM. If Transmission- $K$ holds, your inquiry leads you to knowledge that $\neg$ CDM. Hence, Transmission- $K$ fails. ${ }^{4}$

Crucially, however, this failure is not just supported by an intuition that you do not know that $\neg$ CDM. Rather, we have an independent theoretical argument for this which exploits a couple of platitudinous principles relating the goal of inquiry, question-begging and question-settling, an independently plausible principle relating knowledge and the goal of inquiry, and a highly attractive explanation of a clear difference between Inquiry 1 and 2 in terms of question-begging.

3. Reflection on the structure of question-settling serves to further support this result. To see how, note first that there is a lively and related debate in the literature on whether the following principle holds:

Transmission- $W$. If one has warrant for $p$ and one competently deduces $q$ from $p$, then one thereby has warrant for $q$.

One interesting fact that this debate has unearthed is that the division on Transmission$W$ is driven by a disagreement about the structure of warrant [Pryor 2004]. In particular, we can distinguish between three relevant views: Conservatism [e.g. Wright 2004, 2007, White 2006], Moderatism [e.g. Coliva 2012, 2015] and Liberalism [e.g. Pryor 2000, 2004, Davis 2009]. In the case of perceptual warrants these views disagree about the status of propositions like $\neg \mathrm{CDM}$ for acquiring warrant for propositions like $\mathrm{Z}$ by perceptual means. Conservatives claim that you need an antecedent warrant for propositions like $\neg \mathrm{CDM}$. Moderates claim that, while such propositions need not be antecedently warranted, they must be antecedently assumed in some sense to be specified. In contrast, liberals disagree with both: they deny that propositions like $\neg \mathrm{CDM}$ need be antecedently warranted or assumed. They do grant, however, that they have the potential to defeat one's warrant for propositions

${ }^{4}$ Here is a more detailed statement of the argument:

1. Transmission-K holds. [Assumption for RAA]

2. In Inquiry 2, you know that $\mathrm{Z}$, competently deduce $\neg \mathrm{CDM}$ from $\mathrm{Z}$ and thereupon come to believe that $\neg$ CDM. [Description of Inquiry 2]

3. In Inquiry 2, you come to know that $\neg$ CDM. [1,2]

4. In Inquiry 2, your inquiry into whether $\neg \mathrm{CDM}$ is question-begging. [Question-Begging]

5. In Inquiry 2, your inquiry into whether $\neg \mathrm{CDM}$ doesn't settle the question whether $\neg \mathrm{CDM}$. [4, No Settling]

6. In Inquiry 2, your inquiry into whether $\neg \mathrm{CDM}$ is not successful. [5, Inquiry Success]

7. In Inquiry 2 , your inquiry into $\neg \mathrm{CDM}$ doesn't lead you to knowledge that $\neg \mathrm{CDM}$, i.e. you don't come to know that $\neg$ CDM. [6, Knowledge Sufficiency]

8. $\perp[3,7]$

9. Transmission- $K$ does not hold. $[1,8]$ 
like Z. However, they will do so only in special circumstances such as when one has reason to think that $\neg \mathrm{CDM}$ is true.

Here is how this division bears on the question of Transmission-W. Conservatism and Moderatism license failures of Transmission-W. In Zebra, for instance, they hold that $\neg \mathrm{CDM}$ must already be in place (as either antecedently warranted or assumed) for perceptual means to generate a warrant for Z. As a result, when you reason from $\mathrm{Z}$ to $\neg \mathrm{CDM}$ you effectively fall foul of circular reasoning. Since circular reasoning cannot confer warrant on its conclusion, warrant fails to transmit from $\mathrm{Z}$ to $\neg \mathrm{CDM}$. In contrast, Liberalism does not require $\neg \mathrm{CDM}$ to be in place in any way for you to acquire a warrant for $\mathrm{Z}$ by perceptual means. As a result, the inference from $\mathrm{Z}$ to $\neg \mathrm{CDM}$ will not come out as circular either and is perfectly suited to transmit warrant to the conclusion.

Let's now consider the structure of question-settling. We can easily enough imagine the following analogues to Conservatism, Moderatism and Liberalism about the structure of warrant, which I will label $Q$-Conservatism, $Q$-Moderatism and $Q$ Liberalism respectively. Q-Conservatism claims that in order to settle the question whether $\mathrm{Z}$ by perceptual means, for instance, you need to already have settled the question whether $\neg \mathrm{CDM}$. Q-Moderatism claims that that's too much. All that's needed is that $\neg \mathrm{CDM}$ is already true. Q-Liberalism takes not even this to be required. You can settle the question whether $\mathrm{Z}$ by perceptual means whether or not $\neg \mathrm{CDM}$ has already been settled or whether or not it is already true.

The crucial point is that it is highly plausible that Q-Moderatism is true of the structure of question-settling. Certain facts must already be in place for you to be able to settle the question whether $\mathrm{Z}$ by perceptual means. For instance, when you are in a part of the world that is predominantly populated with cleverly disguised mules, when the animal before you is a cleverly disguised mule, etc., you cannot settle the question whether $\mathrm{Z}$ just by looking. That's why Q-Moderatism is true rather than Q-Liberalism. At the same time, in order to settle the question whether $\mathrm{Z}$ by looking you don't have to already have settled the question of whether the relevant facts are indeed in place. You do not need to already have settled the question of whether you are in cleverly disguised mule county, whether $\neg \mathrm{CDM}$, etc. That would be much too demanding. In fact, if such a requirement were in place, it is hard to see how we could ever settle any question at all. That's why Q-Moderatism is true rather Q-Conservatism.

Given that Moderatism licenses transmission of warrant failures, it should now come as no surprise that question-settling is not transmissive either. In fact, the reason for this is parallel in both cases. Recall that, according to Moderatism, perceptual means will give you a warrant for $\mathrm{Z}$ only if $\neg \mathrm{CDM}$ is assumed. That's why when you deduce $\neg \mathrm{CDM}$ from $\mathrm{Z}$ you fall foul of circular reasoning. Warrant fails to transmit across your deduction. Similarly, settling the question whether $\mathrm{Z}$ by looking requires that you are not in disguised mule county, that $\neg \mathrm{CDM}$, etc. When, having settled $\mathrm{Z}$ by looking, you move on to settle the question whether $\neg \mathrm{CDM}$ by deduction from it being a zebra, you fail in a similar way: you fall foul of begging the question. The property of question-settling fails to transmit across the deduction 
as well.

But, of course, given that knowledge is sufficient for question-settling, this means that in cases in which the property of question-settling fails to transmit across competent deduction, you don't come to know the conclusion of the deduction. Given that, in some such cases (e.g. Inquiry 2), it is independently plausible that you know the premise and that you believe the conclusion based on competent deduction from the premise, this means that Transmission- $K$ is bound to fail. In this way, the argument against Transmission- $K$ receives further support by reflecting on the structure of question-settling.

4. Before moving on, I'd like to point out that we also have what it takes to specify a certain type of case in which Transmission- $K$ fails due to question-begging. To see this, note that the reason why, in Inquiry 2 , your inquiry into whether $\neg \mathrm{CDM}$ begs the question generalises. If Q-Moderatism is true, settling the question whether $p$ in way $W p$ will typically (if not invariably) require that a certain set of facts $\Delta_{W p}$ are already in place. ${ }^{5}$ Anyone who then ventures to settle any further question whether $q$ by deducing, say, $q$ from $p$, where $q$ corresponds to some $i \in \Delta_{W p}$ will fall foul of question-begging. If, in addition, one knows that $p$ by having settled the question whether $p$ in $W p$ and has come to believe $q$ by competent deduction from $p$, we will have a case of Transmission- $K$ failure due to question-begging. We thus have a type of case in which Transmission- $K$ fails due to question-begging. It is this type of case (henceforth The Case) that I will focus on in the remainder of this paper. ${ }^{6}$

5. With the argument against Transmission- $K$ in play, I will now turn to virtue epistemological accounts of knowledge. Recall that my aim here is to show that such accounts have the resources to accommodate not only that Transmission- $K$ fails in the relevant type of case, but also that all remaining instances of Transmission- $K$ continue to hold. First things first, however, I will now sketch the key elements of VE's account of knowledge.

First, VE accepts an account of the normativity of performances with an aim according to which such performances can be assessed along three dimensions:

1. Success. Does the performance attain its aim?

2. Competence. Is the performance produced by the exercise of an ability to attain the aim?

3. Aptness. Is the performance successful because competent?

Second, the relevant notions of ability, its exercise, competent and apt performances are unpacked. Here are rough versions of my own preferred way of doing this [Author 2016]:

\footnotetext{
${ }^{5}$ While I will not discuss the question as to what's in $\Delta_{W p}$ in any detail here, fn. 8 indicates how I aim to tackle the issue.

6 There may be other kinds of case in which Transmission- $K$ fails, perhaps even due to questionbegging. While this may mean that the account of Transmission- $K$ failure is not complete, it does not diminish its significance. After all, understanding only one such kind of case means important progress on the issue.
} 
Abilities. An ability is a way of producing performances that disposes its possessor to attain successes in a certain range when exercised in a set, $\Gamma_{A}$, of suitably favourable conditions.

Exercises of Abilities. An exercise of an ability is a use of the way of producing performances at issue in it.

Competent Performances. A competent performance is a performance that is produced by an exercise of an ability such that the target success is within the range of the ability exercised.

Apt Performances. An apt performance is a successful and competent performance in $\Gamma_{A}$.

On this view, abilities are intrinsically connected to success by the relevant dispositions. However, the strength of the success-connection may vary in a number of ways. For instane, there may be variation in the probability of success conditional on the exercise of ability in $\Gamma_{A}$. The thought here is that the higher the probability of success given the exercise of ability in $\Gamma_{A}$, the stronger the ability (relative to $\Gamma_{A}$ ). Most importantly for present purposes, the strongest abilities (relative to $\Gamma_{A}$ ) feature surefire dispositions, i.e. dispositions such that the exercise of ability in $\Gamma_{A}$ guarantees success. As a result, for abilities featuring surefire dispositions, competent performances in $\Gamma_{A}$ entail successes.

Third, VE takes belief to be one kind of performance with an aim. To be more precise, belief is taken to be a kind of performance with a distinctively epistemic aim. ${ }^{7}$ Accordingly, the account of the normativity of performances applies to belief. Beliefs can be assessed as successful, competent, and apt.

Fourth, VE identifies different core epistemic properties with different normative properties of beliefs as epistemic performances. Most importantly for present purposes, VE identifies justified belief with competent belief and knowledge with apt belief:

VE-JB. One justifiably believes that $p$ if and only if one competently believes that $p$.

VE-K. One knows that $p$ if and only if one aptly believes that $p$.

6. My task is to show that VE bears out failures of Transmission-K in The Case and that it can license instances of Transmission- $K$ in all other cases. In order to achieve this, I will first argue that, given certain assumptions, VE validates an unrestricted version of Transmission-K. This establishes that VE can license instances of Transmission- $K$, including the target ones. Then I will show how champions of VE can replace the assumptions that commit them to Transmission- $K$ in such a way

\footnotetext{
${ }^{7}$ Standardly, this aim is taken to be truth. However, alternatives have also been defended. My own preferred view takes the aim of belief to be knowledge [Author 2016]. Here I want to stay neutral on this issue.
} 
that Transmission- $K$ fails just in The Case. In this way, VE supports just the kind of restricted version of Transmission- $K$ we are after.

To begin with, consider the following bridge principles between Transmission-K and VE-K:

Bridge 1. [C2] and [C3] hold if and only if one believes that $q$ via the exercise of a deductive epistemic ability $\left(\mathrm{DEA}_{q}\right)$.

Bridge 2. [C1] identifies a member of $\Gamma_{D E A q}$.

Now consider the following assumptions:

Assumption 1. The disposition at issue in $\mathrm{DEA}_{q}$ is a surefire disposition.

Assumption 2. There are no other members of $\Gamma_{D E A q}$ besides [C1].

Assumption 3. The range of $\mathrm{DEA}_{q}$ includes all propositions $q$ one may competently deduce from $p$.

VE's framework for the normativity of performances, VE-K, Bridge 1 and 2, and Assumption $1-3$ jointly entail (and thus validate) Transmission-K. Here's why. To begin with, by Bridge 1, Assumption 3 and Competent Performance, if [C2] and [C3] hold, then one will competently believe that $q$. Next, by Bridge 2 and Assumption 2, [C1] specifies the only members $\Gamma_{D E A q}$. Hence, if [C1] holds, the set of conditions to which $\mathrm{DEA}_{q}$ is relative must be satisfied. Hence, if [C1] [C3] hold, then one must also competently believe that $q$ in $\Gamma_{D E A q}$. But now recall that for any ability featuring a surefire disposition, competent performance in $\Gamma_{A}$ entails success. Since, by Assumption 1, the disposition at issue in DEA is a surefire disposition, it follows that if [C1] - [C3] hold, then one must not only competently believe that $q$ in $\Gamma_{D E A q}$ but one's belief that $q$ must also be successful. Thus, by Apt Performance, if [C1] - [C3] hold, one's belief that $q$ is apt. By VE-K, it follows that it qualifies as knowledge, i.e. [C4] holds. Given VE's framework for the normativity of performances, VE-K, Bridge 1 and 2, and Assumption 1-3, we get the result that if $[\mathrm{C} 1]-[\mathrm{C} 3]$ hold, then $[\mathrm{C} 4]$ must hold also. Transmission- $K$ is thus validated.

7. Next, I will show how to accommodate failures of Transmission- $K$ in and just in The Case. Of course, in order to deny Transmission- $K$, champions of VE must block the validation argument from $\S 6$. That means that they will have to resist at least one of the bridge principles or assumptions the argument exploits. At the same time, wherever they may place their resistance, they had better made sure that they do not thereby close the door to explaining that the remaining instances of Transmission- $K$ do hold. It is easy to see that this means that the bridge principles are off limits. And the same goes for Assumption 1. After all, it is needed to ensure that the satisfaction of [C1] - [C3] guarantees the satisfaction of [C4] even for uncontentious instances of Transmission-K. This leaves Assumption 2 and 3. As I am about to argue, both options can be made to work. 
The first and most important step is to connect $\Delta_{W p}$ with VE-K. To see how this can be done, recall that, in the argument against Transmission- $K$, I pointed out that it is uncontentious that knowledge is sufficient for success in inquiry. I also indicated that I have argued elsewhere [Author 2014] that knowledge is not only sufficient but also necessary for success in inquiry. If I am right about this-as, due to limitations of space, I will simply have to assume that I am-and given the plausible assumption that question-settling is also not only sufficient but also necessary for success in inquiry, we get the result that one has settled the question whether $p$ if and only if one aptly believes that $p /$ not- $p$. But in that case, it is immensely plausible that the way of settling the question whether $p$ referred to in The Case, just is an epistemic ability that has $p$ in its range at issue in VE-K. In other words, it is immensely plausible that $W p=E A p$. Moreover, it is also immensely plausible that the set of facts that Q-Moderatism requires to be in place for the question whether $p$ to be settled will just be the set of conditions to which the epistemic ability is relative. In other words, it is also immensely plausible that $\Delta_{W p}=\Gamma_{E A p} \cdot{ }^{8}$ Given that this is so, we can describe The Case in terms of the conceptual resources of VE: (i) one knows that $p$ via the exercise of an epistemic ability, $E A p$ that requires $\Gamma_{E A p}$ to be in place, (ii) one has come to believe that $q$ via the exercise of a deductive epistemic ability, $D E A$, and (iii) $q$ corresponds to some $i \in \Gamma_{E A p}$.

With The Case so described, here's what champions of VE need to do to accommodate the relevant failures of Transmission-K: they need to disallow instances of Transmission- $K$ for any $q$ such that $q$ corresponds to some $i \in \Gamma_{E A p}$. It is not hard to see that there are two ways of achieving this, each corresponding to the denial of one of Assumption 2 and 3. First, pace Assumption 2, champions of VE may maintain that, in addition to one's knowing the premise of one's deduction, a further member of $\Gamma_{D E A q}$ is that $q$ not correspond to any $i \in \Gamma_{E A p}$. Alternatively, pace Assumption 3, they may hold that the range of $\mathrm{DEA}_{q}$ includes only propositions $q$ such that $q$ does not correspond to any $i \in \Gamma_{E A p}$, alongside the requirement that one may competently deduce $q$ from $p$ of course. It is easy to see that (i) either option will accommodate the failure of Transmission- $K$ in The Case, whilst (ii) continuing to allow instances of Transmission- $K$ to hold in all other cases.

8. Does it matter which alternative we opt for? Yes. There is a crucial difference between the two approaches. To see this, let's consider once again the first option, which denies Assumption 2 and holds on to Assumption 3. On this approach, while the belief in the conclusion of the deduction falls short of knowledge, it continues to be justified. The reason for this is that, by Bridge 1, one's belief that $q$ is produced by the exercise of $\mathrm{DEA}_{q}$ and, by Assumption 3, $q$ is within the range of this ability. It follows from Competent Belief that one's belief in $q$ is competent and by VE-JB that it is justified.

In contrast, on the alternative approach, the conclusion belief qualifies as neither

\footnotetext{
${ }^{8}$ In fact, $\Delta_{W p}=\Gamma_{E A p}$ promises to offer a more systematic account of what's in $\Delta_{W p}$. See [Author 2016] for more on how to determine what's in $\Gamma_{E A p}$.
} 
knowledge nor justified. Recall that, here we restrict the range of $\mathrm{DEA}_{q}$ to include only propositions $q$ such that one may competently deduce $q$ from $p$ and $q$ does not correspond to any $i \in \Gamma_{E A p}$. For any $q$ that corresponds to some $i \in \Gamma_{E A p}$, then, while by Bridge 1, one's belief that $q$ may well be produced by the exercise of $\mathrm{DEA}_{q}$, it will not be in the range of this ability. By Competent Belief it is not competent and, by VE-JB, it is not justified.

Finally, it strikes me as highly plausible that, in The Case, the deductive inference not only fails to transmit knowledge, but also justified belief. For instance, when in Inquiry 2, you come to believe $\neg \mathrm{CDM}$ by competent deduction from $\mathrm{Z}$, the question-begging character of your inquiry not only prevents you from coming to know $\neg \mathrm{CDM}$, but also from coming to believe it justifiably. As a result, I take the second option, which places further restrictions on the range of $\mathrm{DEA}_{q}$, to be preferable. This completes the virtue epistemological account of Transmission-K failure I favour.

9. VE is not the only view in the literature that licences failures of Transmission$K$. Most famously, sensitivity accounts of knowledge do so, too. Very roughly, according to sensitivity accounts, one knows that $p$ if and only if one sensitively believes that $p$ [Dretske 1970, Nozick 1981]. And, again very roughly, one's belief that $p$ is sensitive if and only if at the closest worlds at which $p$ false, one does not believe $p$.

It's easy to see that Transmission- $K$ will fail on sensitivity accounts. Just consider Zebra once more. Your belief that $\mathrm{Z}$ is sensitive: at the closest worlds at which $\mathrm{Z}$ false, the enclosure would be empty or you would be standing in front of a different enclosure in which case you'd be looking at some other kind of animal instead. In that case, you would not believe that $Z$. Since your belief that $Z$ is sensitive, sensitivity accounts will predict that you know Z. Now suppose you competently deduce and thereupon come to believe that $\neg \mathrm{CDM}$. Is that belief sensitive as well? No. After all, at the closest worlds at which $\neg \mathrm{CDM}$ false, you'd be looking at a cleverly disguised mule, in which case you'd still believe $\neg \mathrm{CDM}$ (based on your competent deduction from Z). Since your belief that $\neg \mathrm{CDM}$ isn't sensitive, sensitivity accounts will predict that you don't know $\neg$ CDM. Transmission- $K$ fails.

10. What's the relation between the VE approach to Transmission- $K$ and the sensitivity approach? In particular, is there any reason to favour one over the other? As I am about to argue, the answer to this question is yes. What's more, fortunately, there is reason to think that the VE approach is the better one.

To see why, consider first the following case:

Inquiry 3. You are at the zoo. Currently you are standing in front of the zebra enclosure and see a black-and-white striped equine creature inside. Since you can tell a zebra from the way it looks, you come to know that Z. You now want to find out whether $\neg \mathrm{CDM}$. In order to achieve this, you first exploit the equivalence between $\mathrm{Z}$ and $\mathrm{Z} \& \neg \mathrm{CDM}$ to competently deduce $\mathrm{Z} \& \neg \mathrm{CDM}$ and then apply conjunction elimination to arrive at the belief that $\neg \mathrm{CDM}$ [Hawthorne 2004]. 
It's easy to see that the argument from $\S \S 2,3$ that knowledge fails to transmit across competent deduction in Inquiry 2 serves to establish the same result for Inquiry 3. ${ }^{9}$ It's also easy to see that sensitivity accounts can secure this result. After all, your belief that $\neg \mathrm{CDM}$ is no more sensitive here than in the earlier Inquiry 2. Hence, you don't know $\neg \mathrm{CDM}$ here either. So far, so good.

Of course, if knowledge fails to transmit in Inquiry 3, we'd expect it to do so at some specific point in your deduction, i.e. either when you deduce $Z \& \neg C D M$ from $\mathrm{Z}$ or when you deduce $\neg \mathrm{CDM}$ from $\mathrm{Z} \& \neg \mathrm{CDM}$. The question is where exactly this point is. Say you are convinced that Transmission- $K$ fails in cases like Inquiry 2 in which your competent deduction exploits the entailment from $\mathrm{Z}$ to $\neg \mathrm{CDM}$. If so, you will want to say that, in Inquiry 3, knowledge fails to transmit at the first step of the deduction, which exploits the equivalence between $\mathrm{Z}$ and $\mathrm{Z} \& \neg \mathrm{CDM}$. There are at least three reasons for this. First, it seems independently plausible that if you cannot come to know that $\neg \mathrm{CDM}$ via competent deduction from known $\mathrm{Z}$, then the same goes for $\mathrm{Z} \& \neg \mathrm{CDM}$. Second (and relatedly), $\neg \mathrm{CDM}$ is logically weaker than $\mathrm{Z} \& \neg \mathrm{CDM}$. As a result, if knowledge doesn't transmit across competent deduction to $\neg \mathrm{CDM}$, then it would be at least surprising if it did transmit to the stronger $\mathrm{Z} \& \neg \mathrm{CDM}$. Third, the thesis that knowledge is closed under competent conjunction elimination is extremely plausible. Already the stronger principle that if one knows a conjunction, then one knows each conjunct is nearly universally accepted. Rejecting this principle will just be too large of a bullet to bite.

The problem for sensitivity theorists is that they are committed to holding that the deduction fails to transmit at the conjunction elimination step, rather than at the step that exploits the equivalence. The reason for this is that if your belief that $\mathrm{Z}$ is sensitive, then so is your belief that $\mathrm{Z} \& \neg \mathrm{CDM}$ arrived at via competent deduction. After all, the closest worlds at which $\mathrm{Z} \& \neg \mathrm{CDM}$ is false are just the worlds at which $\mathrm{Z}$ is false. According to sensitivity accounts, then, if you know $\mathrm{Z}$ and come to believe that $Z \& \neg C D M$ via competent deduction from $Z$, then you also know that $Z \& \neg C D M$. Sensitivity accounts predict that knowledge does transmit across competent deductions that exploit the equivalence between $\mathrm{Z}$ and $\mathrm{Z} \& \neg \mathrm{CDM}$. So, the problem must lie with the conjunction elimination step. And that's the wrong result.

11. Can the VE approach do better? As a first observation, note that the VE approach does not entail anything about which of the two steps in Inquiry 3 is the problematic one. It only tells us that knowledge does not transmit from $\mathrm{Z}$ to $\neg \mathrm{CDM}$ and hence that at least one step must be problematic. On the upside, this means that the VE approach is at least compatible with the right result here. At the same time, it would be nice to be able to do more than this, i.e. to secure the correct result rather than merely being compatible with it. Fortunately, this can be done also. Here is how.

\footnotetext{
${ }^{9}$ This is unsurprising once it is noted that the only difference between Inquiry 2 and Inquiry 3 is that the competent deduction of $\neg \mathrm{CDM}$ from $\mathrm{Z}$ proceeds via a different route and the above argument simply doesn't depend on the specific route of deduction.
} 
First, the above VE approach only specifies a set of propositions such that knowledge doesn't transmit across competent deduction to members of this set. While this is too coarse-grained for specifying which step in a multi-step deduction is the problematic one, it is all we need when we are dealing with one-step deductions. After all, if the deduction features only one step, then it's clear exactly where transmission failure occurs. For instance, in Inquiry 2, the deduction proceeds in a single step, which exploits the entailment from $\mathrm{Z}$ to $\neg \mathrm{CDM}$. Here, it's clear that this is where transmission failure occurs.

While it would be nice to have a fully general account of just where knowledge fails to transmit in multi-step deductions, I must confess that I don't have one. However, I have the following proposal for a partial account:

Transmission Failure. If knowledge fails to transmit across a competent deduction that exploits a certain logical relationship between the members of a certain set of propositions, then it also fails to transmit across any competent deduction that exploits an a priori equivalent logical relationship between the members of this set.

For instance, if knowledge fails to transmit across any competent deduction that exploits the entailment from $p$ to $q$, then it also fails to transmit across any competent deduction that exploits a logical relationship a priori equivalent to the entailment from $p$ to $q$. This much seems plausible enough.

Now, we have already seen that knowledge fails to transmit from competent deduction from $\mathrm{Z}$ to $\neg \mathrm{CDM}$ in Inquiry 2 . We have also seen that, in Inquiry 2 , the deduction is a one-step deduction, which exploits the entailment from $\mathrm{Z}$ to $\neg \mathrm{CDM}$. This means that the deduction fails at the step that exploits the entailment. Crucially, the entailment from $\mathrm{Z}$ to $\neg \mathrm{CDM}$ is a priori equivalent to the equivalence between $\mathrm{Z}$ and $\mathrm{Z} \& \neg \mathrm{CDM}$, i.e. $(\mathrm{Z} \rightarrow \neg \mathrm{CDM}) \equiv(\mathrm{Z} \equiv(\mathrm{Z} \& \neg \mathrm{CDM}))$. By Transmission Failure, it follows that knowledge fails to transmit across any competent deduction that exploits the equivalence between $\mathrm{Z}$ and $\mathrm{Z} \& \neg \mathrm{CDM}$. In this way, the $\mathrm{VE}$ approach can secure the desired result.

12. In this paper I have done three things. First, I have mounted a case against Transmission- $K$, which not only provided theoretical reason to believe that this principle is false but also specified a certain type of case in which it fails. Second, I have shown that champions of a certain type of virtue epistemological account of knowledge can countenance a suitably restricted version of Transmission- $K$, which licences failures of transmission in the problematic type of case, whilst allowing for knowledge to transmit in all other cases. Finally, third, I have compared this virtue epistemological approach to the most prominent competitor in the literature, which explains transmission failures in terms of a sensitivity account of knowledge. I have argued that the virtue epistemological approach is favourable as only it can pin failures of Transmission- $K$ to the right step in all relevant cases. This paper has thus brought good news for deniers of Transmission- $K$ whom it provides with further support for their view as well as for virtue epistemologists who can success- 
fully solve another difficult epistemological problem and score points against the competition.

\section{References}

Author 2013. Author's work.

Author 2014. Author's work.

Author 2016. Author's work.

Coliva, A. 2012. Varieties of failure (of warrant transmission: what else?!). Synthese, 189:335-354.

Coliva, A. 2015. Extended Rationality. A Hinge Epistemology. Palgrave Macmillan, Basingstoke.

Davidson, D. 2005. Truth, Language and History. Clarendon Press, Oxford.

Davis, M. 2009. Two purposes of arguing and two epistemic projects. In Ravenscroft, I., editor, Minds, Ethics, and Conditionals: Themes from the Philosophy of Frank Jackson. Oxford University Press, Oxford.

Dretske, F. 1970. Epistemic operators. Journal of Philosophy, 67:1007-23.

Feldman, R. 2002. Epistemological duties. In Moser, P., editor, The Oxford Handbook of Epistemology. Oxford University Press, New York.

Greco, J. 2010. Achieving Knowledge. Cambridge University Press, Cambridge.

Greco, J. 2012. A (different) virtue epistemology. Philosophy and Phenomenological Research, 85:1-26.

Hawthorne, J. 2004. Knowledge and Lotteries. Oxford University Press, Oxford.

Hieronymi, P. 2009. Two kinds of agency. In O'Brien, L. and Soteriou, M., editors, Mental Actions. Oxford University Press, Oxford.

Kvanvig, J. 2003. The Value of Knowledge and the Pursuit of Understanding. Cambridge University Press, Cambridge.

Lynch, M. 2005. True To Life. Why Truth Matters. MIT Press, Cambridge/MA.

Markie, P. 2005. Easy knowledge. Philosophy and Phenomenological Research, 70:406-16.

Millar, A. 2011. Why knowledge matters. Proceedings of the Aristotelian Society, Suppl. Vol., 85:63-81.

Nozick, R. 1981. Philosophical Explanations. Oxford University Press, Oxford. 
Pritchard, D. 2007. How to be a neo-Moorean. In Goldberg, S., editor, Internalism and Externalism in Semantics and Epistemology. Oxford University Press, Oxford.

Pryor, J. 2000. The skeptic and the dogmatist. Nô̂s, (34):517-49.

Pryor, J. 2004. What's wrong with Moore's argument? Philosophical Issues, 14:349-78.

Rorty, R. 1995. Is truth a goal of enquiry? Davidson vs. Wright. The Philosophical Quarterly, 45:281-300.

Sosa, E. 2011. Knowing Full-Well. Princeton University Press, Princeton/NJ.

Sosa, E. 2015. Judgment and Agency. Oxford University Press, Oxford.

White, R. 2006. Problems for dogmatism. Philosophical Studies, 131:525-57.

Williamson, T. 2000. Knowledge and Its Limits. Oxford University Press, Oxford.

Wright, C. 2004. Warrant for nothing (and foundations for free)? Proceedings of the Aristotelian Society, Suppl. Vol., 78:167-212.

Wright, C. 2007. The perils of dogmatism. In Nuccetelli, S., editor, Themes from G.E. Moore. New Essays in Epistemology and Ethics. Oxford University Press, Oxford. 